\title{
STRONGLY STARLIKE FUNCTIONS ASSOCIATED WITH THE DZIOK-SRIVASTAVA OPERATOR
}

\author{
JIN-LIN LIU
}

Abstract. In terms of the Dziok-Srivastava operator, we introduce and study some new classes of strongly starlike functions. Certain properties of these subclasses are studied.

\section{Introduction}

Let $A$ denote the class of functions $f(z)$ of the form

$$
f(z)=z+\sum_{n=2}^{\infty} a_{n} z^{n}
$$

which are analytic in the unit $\operatorname{disc} E=\{z:|z|<1\}$. A function $f(z)$ belonging to the class $A$ is said to be convex of order $\gamma$ in $E$ if and only if

$$
\operatorname{Re}\left\{1+\frac{z f^{\prime \prime}(z)}{f^{\prime}(z)}\right\}>\gamma \quad(z \in E ; 0 \leq \gamma<1) .
$$

A function $f(z)$ belonging to the class $A$ is said to be starlike of order $\gamma$ in $E$ if and only if

$$
\operatorname{Re}\left\{\frac{z f^{\prime}(z)}{f(z)}\right\}>\gamma \quad(z \in E ; 0 \leq \gamma<1) .
$$

We denote by $C(\gamma)$ the class of all functions in $A$ which are convex of order $\gamma$ in $E$ and by $S^{*}(\gamma)$ the class of all functions in $A$ which are starlike of order $\gamma$ in $E$. From (1.2) and (1.3), we can see that $f(z) \in C(\gamma)$ if and only if $z f^{\prime}(z) \in S^{*}(\gamma)$.

If $f(z) \in A$ satisfies

$$
\left|\arg \left(\frac{z f^{\prime}(z)}{f(z)}-\gamma\right)\right|<\frac{\pi}{2} \beta \quad(z \in E ; 0<\beta \leq 1,0 \leq \gamma<1),
$$

Received March 15, 2002; revised October 9, 2002.

2000 Mathematics Subject Classification. Primary. 30C45, 26A33; Secondary. 33C20.

Key words and phrases. The Dziok-Srivastava operator, strongly starlike function, strongly convex function. 
then we say that $f(z)$ is strongly starlike of order $\beta$ and type $\gamma$ in $E$, and we denote by $S^{*}(\beta, \gamma)$ the class of all such functions. If $f(z) \in A$ satisfies

$$
\left|\arg \left(1+\frac{z f^{\prime \prime}(z)}{f^{\prime}(z)}-\gamma\right)\right|<\frac{\pi}{2} \beta \quad(z \in E ; 0<\beta \leq 1,0 \leq \gamma<1),
$$

then $f(z)$ is said to be strongly convex of order $\beta$ and type $\gamma$ in $E$, and denote by $f(z) \in$ $C(\beta, \gamma)$. It is obvious that $f(z) \in A$ belongs to $C(\beta, \gamma)$ if and only if $z f^{\prime}(z) \in S^{*}(\beta, \gamma)$. Further, we note that $S^{*}(1, \gamma)=S^{*}(\gamma)$ and $C(1, \gamma)=C(\gamma)$.

Very recently, Dziok and Srivastava [3] have given a systematic investigation of a linear operator $H\left(\alpha_{1}, \alpha_{2}, \ldots, \alpha_{q} ; \beta_{1}, \beta_{2}, \ldots, \beta_{s}\right)$ which is defined by the generalized hypergeometric function (see, for details, [3]). They showed that

$$
\begin{aligned}
& z H^{\prime}\left(\alpha_{1}, \alpha_{2}, \ldots, \alpha_{q} ; \beta_{1}, \beta_{2}, \ldots, \beta_{s}\right) f(z) \\
= & \alpha_{1} H\left(\alpha_{1}+1, \alpha_{2}, \ldots, \alpha_{q} ; \beta_{1}, \beta_{2}, \ldots, \beta_{s}\right) f(z)-\left(\alpha_{1}-1\right) H\left(\alpha_{1}, \alpha_{2}, \ldots, \alpha_{q} ; \beta_{1}, \beta_{2}, \ldots, \beta_{s}\right) f(z)
\end{aligned}
$$

Let $q, s \in N, 0<\beta \leq 1$ and $0 \leq \gamma<1$. We now introduce the following classes in terms of the linear operator $H\left(\alpha_{1}, \alpha_{2}, \ldots, \alpha_{q} ; \beta_{1}, \beta_{2}, \ldots, \beta_{s}\right)$ :

$$
\begin{aligned}
& M_{\alpha_{1}}(\beta, \gamma)=\left\{f(z) \in A: H_{q, s}\left(\alpha_{1}\right) f(z) \in S^{*}(\beta, \gamma), \frac{z\left(H_{q, s}\left(\alpha_{1}\right) f(z)\right)^{\prime}}{H_{q, s}\left(\alpha_{1}\right) f(z)} \neq \gamma \quad \text { for } z \in E\right\} \\
& \text { and } \\
& P_{\alpha_{1}}(\beta, \gamma)=\left\{f(z) \in A: H_{q, s}\left(\alpha_{1}\right) f(z) \in C(\beta, \gamma), 1+\frac{z\left(H_{q, s}\left(\alpha_{1}\right) f(z)\right)^{\prime \prime}}{\left(H_{q, s}\left(\alpha_{1}\right) f(z)\right)^{\prime}} \neq \gamma \text { for } z \in E\right\},
\end{aligned}
$$

where, for convenience,

$$
H_{q, s}\left(\alpha_{1}\right)=H\left(\alpha_{1}, \alpha_{2}, \ldots, \alpha_{q} ; \beta_{1}, \beta_{2}, \ldots, \beta_{s}\right) .
$$

For $q=s+1$ and $\alpha_{1}=1, \alpha_{2}=\beta_{1}, \ldots, \alpha_{q}=\beta_{s}$, it is easy to see that $M_{1}(\beta, \gamma)$ is the class of strongly starlike functions of order $\beta$ and type $\gamma$ whereas $P_{1}(\beta, \gamma)$ is the class of strongly convex functions of order $\beta$ and type $\gamma$. Further, when $\beta=1$, we note that $M_{1}(1, \gamma)=S^{*}(\gamma)$ and $P_{1}(1, \gamma)=C(\gamma)$

In this note, we shall study some inclusion properties of the classes $M_{\alpha_{1}}(\beta, \gamma)$ and $P_{\alpha_{1}}(\beta, \gamma)$. The basic tool of our investigation is the following lemma due to Nunokawa $[16,17]$.

Lemma. Let a function $p(z)=1+b_{1} z+\cdots$ be analytic in $E$ and $p(z) \neq 0(z \in E)$. If there exists a point $z_{0} \in E$ such that

$$
|\arg p(z)|<\pi \beta / 2\left(|z|<\left|z_{0}\right|\right) \quad \text { and }\left|\arg p\left(z_{0}\right)\right|=\pi \beta / 2(0<\beta \leq 1),
$$

then we have $z_{0} p^{\prime}\left(z_{0}\right) / p\left(z_{0}\right)=i k \beta$, where

$$
\begin{aligned}
& k \geq \frac{1}{2}\left(a+\frac{1}{a}\right) \quad\left(\text { where } \arg p\left(z_{0}\right)=\pi \beta / 2\right), \\
& k \leq-\frac{1}{2}\left(a+\frac{1}{a}\right) \quad\left(\text { where } \arg p\left(z_{0}\right)=-\pi \beta / 2\right),
\end{aligned}
$$


and $\left(p\left(z_{0}\right)\right)^{1 / \beta}= \pm i a(a>0)$.

\section{Main Results}

Our first inclusion theorem is the following

Theorem 1. $M_{\alpha_{1}+1}(\beta, \gamma) \subset M_{\alpha_{1}}(\beta, \gamma)$ for $\alpha_{1}>1-\gamma$ and $0 \leq \gamma<1$.

Proof. Let $f(z) \in M_{\alpha_{1}+1}(\beta, \gamma)$. Suppose that

$$
\frac{z\left(H_{q, s}\left(\alpha_{1}\right) f(z)\right)^{\prime}}{H_{q, s}\left(\alpha_{1}\right) f(z)}=\gamma+(1-\gamma) p(z)
$$

where $p(z)=1+c_{1} z+c_{2} z^{2}+\cdots$ is analytic in $E$ and $p(z) \neq 0$ for all $z \in E$. By (2.1) and (1.6), we have

$$
\begin{aligned}
\frac{H_{q, s}\left(\alpha_{1}+1\right) f(z)}{H_{q, s}\left(\alpha_{1}\right) f(z)} & =\frac{1}{\alpha_{1}}\left[\frac{z\left(H_{q, s}\left(\alpha_{1}\right) f(z)\right)^{\prime}}{H_{q, s}\left(\alpha_{1}\right) f(z)}+\left(\alpha_{1}-1\right)\right] \\
& =\frac{1}{\alpha_{1}}\left[(1-\gamma) p(z)+\left(\gamma+\alpha_{1}-1\right)\right] .
\end{aligned}
$$

Differentiating both sides of (2.2) logarithmically, it follows that

or

$$
\begin{aligned}
\frac{z\left(H_{q, s}\left(\alpha_{1}+1\right) f(z)\right)^{\prime}}{H_{q, s}\left(\alpha_{1}+1\right) f(z)} & =\frac{z\left(H_{q, s}\left(\alpha_{1}\right) f(z)\right)^{\prime}}{H_{q, s}\left(\alpha_{1}\right) f(z)}+\frac{(1-\gamma) z p^{\prime}(z)}{(1-\gamma) p(z)+\left(\gamma+\alpha_{1}-1\right)} \\
& =(1-\gamma) p(z)+\gamma+\frac{(1-\gamma) z p^{\prime}(z)}{(1-\gamma) p(z)+\left(\gamma+\alpha_{1}-1\right)}
\end{aligned}
$$

$$
\frac{z\left(H_{q, s}\left(\alpha_{1}+1\right) f(z)\right)^{\prime}}{H_{q, s}\left(\alpha_{1}+1\right) f(z)}-\gamma=(1-\gamma) p(z)+\frac{(1-\gamma) z p^{\prime}(z)}{(1-\gamma) p(z)+\left(\gamma+\alpha_{1}-1\right)} .
$$

Suppose that there exists a point $z_{0} \in E$ such that

$$
|\arg p(z)|<\pi \beta / 2\left(|z|<\left|z_{0}\right|\right) \quad \text { and } \quad\left|\arg p\left(z_{0}\right)\right|=\pi \beta / 2 .
$$

Then, by Lemma, we can write that $z_{0} p^{\prime}\left(z_{0}\right) / p\left(z_{0}\right)=i k \beta$ and $\left(p\left(z_{0}\right)\right)^{1 / \beta}= \pm i a(a>0)$.

Therefore, if $\arg p\left(z_{0}\right)=\pi \beta / 2$, then

$$
\begin{aligned}
\frac{z\left(H_{q, s}\left(\alpha_{1}+1\right) f\left(z_{0}\right)\right)^{\prime}}{H_{q, s}\left(\alpha_{1}+1\right) f\left(z_{0}\right)}-\gamma & =(1-\gamma) p\left(z_{0}\right)\left[1+\frac{z_{0} p^{\prime}\left(z_{0}\right) / p\left(z_{0}\right)}{(1-\gamma) p\left(z_{0}\right)+\left(\gamma+\alpha_{1}-1\right)}\right] \\
& =(1-\gamma) a^{\beta} e^{i \pi \beta / 2}\left[1+\frac{i k \beta}{(1-\gamma) a^{\beta} e^{i \pi \beta / 2}+\left(\gamma+\alpha_{1}-1\right)}\right] .
\end{aligned}
$$

This implies that

$$
\arg \left\{\frac{z_{0}\left(H_{q, s}\left(\alpha_{1}+1\right) f\left(z_{0}\right)\right)^{\prime}}{H_{q, s}\left(\alpha_{1}+1\right) f\left(z_{0}\right)}-\gamma\right\}
$$


$=\frac{\pi}{2} \beta+\arg \left\{1+\frac{i k \beta}{(1-\gamma) a^{\beta} e^{i \pi \beta / 2}+\left(\gamma+\alpha_{1}-1\right)}\right\}$

$=\frac{\pi}{2} \beta+\tan ^{-1}\left\{\frac{k \beta\left[\left(\gamma+\alpha_{1}-1\right)+(1-\gamma) a^{\beta} \cos \left(\frac{\pi \beta}{2}\right)\right]}{\left(\gamma+\alpha_{1}-1\right)^{2}+2\left(\gamma+\alpha_{1}-1\right)(1-\gamma) a^{\beta} \cos \left(\frac{\pi \beta}{2}\right)+(1-\gamma)^{2} a^{2 \beta}+k \beta(1-\gamma) a^{\beta} \sin \left(\frac{\pi \beta}{2}\right)}\right\}$

$\geq \frac{\pi}{2} \beta \quad\left(\right.$ where $\left.k \geq \frac{1}{2}\left(a+\frac{1}{a}\right)>1\right)$,

which contradicts the hypothesis that $f(z) \in M_{\alpha_{1}+1}(\beta, \gamma)$.

Similarly, if $\arg p\left(z_{0}\right)=-\pi \beta / 2$, then we obtain that

$$
\arg \left\{\frac{z_{0}\left(H_{q, s}\left(\alpha_{1}+1\right) f\left(z_{0}\right)\right)^{\prime}}{H_{q, s}\left(\alpha_{1}+1\right) f\left(z_{0}\right)}-\gamma\right\} \leq-\frac{\pi}{2} \beta,
$$

which also contradicts the hypothesis that $f(z) \in M_{\alpha_{1}+1}(\beta, \gamma)$.

Thus the function $p(z)$ has to satisfy $|\arg p(z)|<\pi \beta / 2(z \in E)$. This shows that

$$
\left|\arg \left\{\frac{z\left(H_{q, s}\left(\alpha_{1}\right) f(z)\right)^{\prime}}{H_{q, s}\left(\alpha_{1}\right) f(z)}-\gamma\right\}\right| \leq \frac{\pi}{2} \beta \quad(z \in E) .
$$

This evidently completes the proof of Theorem 1.

By Theorem 1, we also have the following

Theorem 2. $P_{\alpha_{1}+1}(\beta, \gamma) \subset P_{\alpha_{1}}(\beta, \gamma)$ for $\alpha_{1}>1-\gamma$ and $0 \leq \gamma<1$.

Proof. $\quad f(z) \in P_{\alpha_{1}+1}(\beta, \gamma) \Leftrightarrow H_{q, s}\left(\alpha_{1}+1\right) f(z) \in C(\beta, \gamma)$

$$
\begin{aligned}
& \Leftrightarrow z\left(H_{q, s}\left(\alpha_{1}+1\right) f(z)\right)^{\prime} \in S^{*}(\beta, \gamma) \Leftrightarrow H_{q, s}\left(\alpha_{1}+1\right)\left(z f^{\prime}(z)\right) \in S^{*}(\beta, \gamma) \\
& \Leftrightarrow z f^{\prime}(z) \in M_{\alpha_{1}+1}(\beta, \gamma) \Rightarrow z f^{\prime}(z) \in M_{\alpha_{1}}(\beta, \gamma) \\
& \Leftrightarrow H_{q, s}\left(\alpha_{1}\right)\left(z f^{\prime}(z)\right) \in S^{*}(\beta, \gamma) \Leftrightarrow z\left(H_{q, s}\left(\alpha_{1}\right) f(z)\right)^{\prime} \in S^{*}(\beta, \gamma) \\
& \Leftrightarrow H_{q, s}\left(\alpha_{1}\right) f(z) \in C(\beta, \gamma) \Leftrightarrow f(z) \in P_{\alpha_{1}}(\beta, \gamma) .
\end{aligned}
$$

We next state.

Theorem 3. Let $v>-\gamma$ and $0 \leq \gamma<1$. If $f(z) \in M_{\alpha_{1}}(\beta, \gamma)$ with $z\left(H_{q, s}\left(\alpha_{1}\right) J_{v} f(z)\right)^{\prime} /$ $H_{q, s}\left(\alpha_{1}\right) J_{v} f(z) \neq \gamma$ for all $z \in E$, then $J_{v} f(z) \in M_{\alpha_{1}}(\beta, \gamma)$, where $J_{v} f(z)$ is given by

$$
J_{v} f(z)=\frac{v+1}{z^{v}} \int_{0}^{z} t^{v-1} f(t) d t \quad(v>-1 ; f(z) \in A) .
$$

Proof. Let

$$
\frac{z\left(H_{q, s}\left(\alpha_{1}\right) J_{v} f(z)\right)^{\prime}}{H_{q, s}\left(\alpha_{1}\right) J_{v} f(z)}=\gamma+(1-\gamma) p(z)
$$

where $p(z)$ is analytic in $E, p(0)=1$ and $p(z) \neq 0(z \in E)$. From (2.3), we have

$$
z\left(H_{q, s}\left(\alpha_{1}\right) J_{v} f(z)\right)^{\prime}=(v+1) H_{q, s}\left(\alpha_{1}\right) f(z)-v H_{q, s}\left(\alpha_{1}\right) J_{v} f(z) .
$$


By (2.4) and (2.5), we obtain

$$
\frac{H_{q, s}\left(\alpha_{1}\right) f(z)}{H_{q, s}\left(\alpha_{1}\right) J_{v} f(z)}=\frac{1}{v+1}[(1-\gamma) p(z)+(\gamma+v)] .
$$

Differentiating (2.6) logarithmically, we have

$$
\frac{z\left(H_{q, s}\left(\alpha_{1}\right) f(z)\right)^{\prime}}{H_{q, s}\left(\alpha_{1}\right) f(z)}-\gamma=(1-\gamma) p(z)+\frac{(1-\gamma) z p^{\prime}(z)}{(1-\gamma) p(z)+(\gamma+v)} .
$$

The remaining part of the proof is similar to that of Theorem 1 and so is omitted.

Finally, we derive the following.

Theorem 4. Let $v>-\gamma$ and $0 \leq \gamma<1$. If $f(z) \in P_{\alpha_{1}}(\beta, \gamma)$ and $1+z\left(H_{q, s}\left(\alpha_{1}\right) J_{v} f(z)\right)^{\prime \prime} /$ $\left(H_{q, s}\left(\alpha_{1}\right) J_{v} f(z)\right)^{\prime} \neq \gamma$ for all $z \in E$, then $J_{v} f(z) \in P_{\alpha_{1}}(\beta, \gamma)$.

Proof.

$$
\begin{aligned}
& f(z) \in P_{\alpha_{1}}(\beta, \gamma) \Leftrightarrow z f^{\prime}(z) \in M_{\alpha_{1}}(\beta, \gamma) \\
\Leftrightarrow & J_{v}\left(z f^{\prime}(z)\right) \in M_{\alpha_{1}}(\beta, \gamma) \Leftrightarrow z\left(J_{v} f(z)\right)^{\prime} \in M_{\alpha_{1}}(\beta, \gamma) \\
\Leftrightarrow & J_{v} f(z) \in P_{\alpha_{1}}(\beta, \gamma) .
\end{aligned}
$$

\section{Acknowledgement}

The author would like to express his gratitude to the referee for many valuable advices.

\section{References}

[1] S. D. Bernardi, Convex and starlike univalent functions, Trans. Amer. Math. Soc. 135(1969), 429-446.

[2] B. C. Carlson and D. B. Shaffer, Starlike and prestarlike hypergeometric functions, SIAM J. Math. Anal. 15(1984), 737-745.

[3] J. Dziok and H. M. Srivastava, Classes of analytic functions associated with the generalized hypergeometric function, Appl. Math. Comput. 103(1999), 1-13.

[4] A. Gangaharan and V. Ravichandran, Radii of convexity and strong starlikeness for some classes of analytic functions, J. Math. Anal. Appl. 211(1997), 301-313.

[5] Yu. E. Hohlov, Operators and operations in th class of univalent functions, IZV. Vyšs. Učebn. Zaved. Mathematika 10(1978), 83-89.

[6] I. B. Jung, Y. C. Kim and H. M. Srivastava, The Hardy space of analytic functions associated with certain one-parameter families of integral operators, J. Math. Anal. Appl. 176(1993), 138-147.

[7] Y. C. Kim, S. H. Lee and H. M. Srivastava, Some properties of convolution operators in the class $P_{\alpha}(\beta)$, J. Math. Anal. Appl. 187(1994), 498-512.

[8] R. J. Libera, Some classes of regular univalent functions, Proc. Amer. Math. Soc. 16(1965), 755-758.

[9] J. L. Liu, Note on the Ruscheweyh derivatives, J. Math. Anal. Appl. 199(1996), 936-940. 
[10] J. L. Liu, Noor integral operator and strongly starlike functions, J. Math. Anal. Appl. 261(2001), 441-447.

[11] A. E. Livingston, On the radius of univalence of certain analytic functions, Proc. Amer. Math. Soc. 17(1966), 352-357.

[12] W. Ma and D. Minda, Coefficient inequalities for strongly close-to-convex functions, J. Math. Anal. Appl. 205(1997), 537-553.

[13] S. Ruscheweyh, New criteria for univalent functions, Proc. Amer. Math. Soc. 49(1975), 109-115.

[14] H. M. Srivastava and S. Owa, Some characterization and distortion theorems involving fractional calculus, generalized hypergeometric functions, Hadamard products, linear operators, and certain subcalsses of analytic functions, Nagoya Math. J. 106(1987), 1-28.

[15] H. M. Srivastava and S. Owa (Eds.), Univalent Functions, Fractional Calculus, and Their Applications, Halsted Press (Ellis Horwood Limited, Chichester), Wiley, New York/Chichester/Brisbane/Toronto, 1989.

[16] M. Nunokawa, On properties of non-caratheodory functions, Proc. Japan Acad. Ser. A Math. Sci. 68(1992), 152-153.

[17] M. Nunokawa et al., Some results for strongly starlike functions, J. Math. Anal. Appl. 212(1997), 98-106.

Department of Mathematics, Yangzhou University, Yangzhou 225002, Jiangsu, People's Republic of China.

E-mail: jlliucn@yahoo.com.cn 